\title{
Migrant charging in the NHS: how doctors can support patients when hospital care is denied
}

This Feature (BMJ 2019;365:12281, doi:10.1136/bmj.12281) has an error in the fifth paragraph of the section titled "Risks of discrimination." The hospital trust in which 541 women were charged for their maternity care was Lewisham and Greenwich NHS Trust, not Luton and Dunstable University Hospital as stated. 